\title{
Optimal passivity design of a virtual coupling including FIR-type fractional derivatives for a haptic interface
}

\author{
Masayuki Kawai ${ }^{1, *}$, Takuya Yamagishi ${ }^{1}$, and Shouta Minami ${ }^{1}$ \\ ${ }^{1}$ University of Fukui, Department of Mechanical Engineering, 3-9-1 Fukui, 9108507, Japan
}

\begin{abstract}
This paper describes a haptic interface with a virtual coupling, including fractional derivatives. A haptic interface is a force feedback technology in virtual reality that takes advantage of the human sense of touch. In a haptic interface, virtual impedance, that is called virtual coupling (VC), is commonly used between the virtual and real objects to calculate reaction force. VC generally consists of a virtual stiffness and damper, but the stiffness has to be set low in a system with long sampling periods. In order to increase the virtual stiffness, this paper considers a VC including fractional derivatives approximated by an FIR approximation. First, we theoretically analyse effects of a single fractional derivative term by using the passivity analysis, but the result shows that its effects depend on the occurred frequency. This paper, therefore, proposes a method to combine multiple fractional derivative terms in a $\mathrm{VC}$ and a method to optimize parameters in each fractional derivative term. Finally, experiments are performed to measure the maximum value of the stiffness to illustrate the effects of the proposed method.
\end{abstract}

\section{Introduction}

A haptic interface is a force feedback technology in virtual reality that takes advantage of the human sense of touch. Operators can intuitively feel and manipulate virtual objects in a virtual environment. In the haptic interface, virtual impedance called "virtual coupling (VC)" is designed between the virtual and real objects, and the force that the operator senses is calculated from the impedance. A common VC generally consists of a virtual stiffness and virtual damper. In a system with long sampling periods, however, the stiffness has to be set low for the stability, and the system only presents virtual objects with soft surfaces. The limitations of virtual stiffness have been examined using passivity analysis [1-4]. Various devices and methods have also been studied for increasing the stiffness [5-8]. On the other hand, applications of fractional derivatives [9] to control methods or model physical phenomena have also been recently studied [10-17]. A fractional derivative is a

\footnotetext{
* Corresponding author: m_kawai@u-fukui.ac.jp
} 
generalization of an ordinary derivative to a non-integer order. When a fractional derivative is used in control methods, it can easily describe the intermediate effects between ordinal integer-order derivatives.

This research studies the effects of a VC including a fractional derivative, approximated by a finite impulse response (FIR approximation) [13, 14]. An FIR approximation is a less precise approximation than the other types of approximation [15-17], but is the easiest type of approximation to be designed, in which the order of the derivative and filter order should only be fixed. First, this paper considers the effect of a VC including a single term of a fractional derivative theoretically, and derives that its effects depend on the occurred frequency. Then, this paper proposes a method to use multiple terms of a fractional derivative and optimize parameters on each term. Finally, experiments are performed to measure the maximum value of the stiffness to illustrate the effects of the proposed method.

\section{Background}

\subsection{Haptic interface and a common PDVC}

In an impedance display in a haptic interface, the force sensed by an operator is calculated using a VC that typically consists of a virtual stiffness and damper. Consider a Pen-type haptic display system and a one-degree-of-freedom modelling, as shown in Fig. 1,

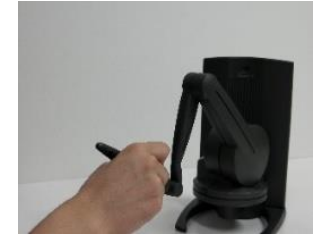

(a) Example of a haptic interface

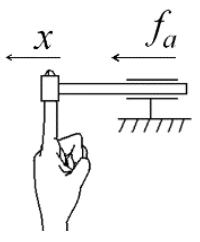

(b) 1-DOF Modeling of a haptic interface

Fig. 1. Haptic display system

where $x(\mathrm{~m})$ is the fingertip position and $f_{a}(\mathrm{~N})$ is the force exerted by the device. The ordinary virtual coupling impedance for a virtual wall at $x=0$ is given by

$$
f_{a}=K x+B \frac{d x}{d t}
$$

where $K$ and $B$ are the coefficients of the virtual stiffness and damper, respectively. As (1) is the same as a common PD feedback control; thus, we call it 'PDVC' herein. In a haptic interface, it is difficult to use the usual stability analysis because it involves human dynamics that are unknown. Therefore, passivity analysis is commonly used instead. Colgate et al. [3][4] have derived the passivity condition for this system. A necessary and sufficient condition for the system except the operator's dynamics to become passive is represented by

$$
b>\frac{T}{2} \frac{1}{1-\cos \omega T} \operatorname{Re}\left\{\left(1-e^{-j \omega T}\right) H\left(e^{j \omega T}\right)\right\},
$$

where $b$ is the viscous resistance of the device, $T$ is the sampling period, $H(z)$ is a transfer function of the virtual environment and $\omega$ is the frequency that satisfies $0 \leq \omega \leq \omega_{N}$, where $\omega_{N}$ is the Nyquist frequency. For the PDVC case, inequality (2) becomes

$$
b>\frac{K T}{2}-B \cos \omega T \text {. }
$$


Equation (3) shows that the maximum value of $K$ depends on $\omega$. In a system with long sampling periods, $K$ has to be set low and the system can display only virtual objects with soft surfaces.

\subsection{Fractional derivatives}

A fractional derivative is a calculus used to generalize the ordinary derivative into a noninteger order, which allows us to utilize the $0.5^{\text {th }}$ or $1.5^{\text {th }}$ order derivatives. This research uses an FIR approximation that transforms a fractional derivative in the Grünwald-Letnikov form directly to the discrete time domain. The form is described by

$$
D^{\alpha} x(t)=\left(\frac{d}{d t}\right)^{\alpha} x(t)=\lim _{h \rightarrow 0} \frac{1}{h^{\alpha}} \sum_{m=0}^{\infty} \frac{(-1)^{m} \Gamma(\alpha+1)}{\Gamma(m+1) \Gamma(\alpha-m+1)} x(t-m h),
$$

where $\alpha$ is the derivative order, which is a non-integer number. $D^{\alpha}$ represents the $\alpha$ th-order derivative and $\Gamma$ is the gamma function. In the FIR approximation, the equation is directly transferred into the discrete time domain with a finite filter order $n$ as

$$
Z\left\{D^{\alpha} x(t)\right\}=\frac{1}{T^{\alpha}} \sum_{m=0}^{n} \frac{(-1)^{m} \Gamma(\alpha+1)}{\Gamma(m+1) \Gamma(\alpha-m+1)} Z^{-m} .
$$

\section{Virtual coupling with a single term of a fractional derivative}

\subsection{VC with a single term of a fractional derivative}

This paper considers the replacement of the virtual damper in a PDVC with a fractional derivative. There is a possibility that a VC with fractional derivative feedback makes the virtual stiffness higher because it can use derivatives greater than those of the first order. First, this research uses a new VC which replaces the virtual damper in (1) as follows:

$$
f_{a}=K x+B_{\alpha} \frac{d^{\alpha} x}{d t^{\alpha}}
$$

The first term on the right side of this equation is the same virtual stiffness as in the PDVC. The second term represents the fractional derivative feedback. This method requires the determination of two parameters, i.e., the derivative order $\alpha$ and filter order $n$ of the approximation. This definition is also a generalization of PDVC; obviously, the case of $\alpha=1$ corresponds to a PDVC. To distinguish this definition from a PDVC, we call it a ' $\mathrm{PD}^{\alpha} \mathrm{VC}$ ' herein. With the definition (5), the transfer function of a $\mathrm{PD}^{\alpha} \mathrm{VC}$ is described by

$$
H(z)=K+\frac{B_{\alpha}}{T^{\alpha}} \sum_{m=0}^{n} \frac{(-1)^{m} \Gamma(\alpha+1)}{\Gamma(m+1) \Gamma(\alpha-m+1)} z^{-m} .
$$

By substituting (7) into (2), the passivity condition becomes

$$
b>\frac{K T}{2}-\frac{B_{\alpha}}{2 T^{\alpha-1}(1-\cos \omega T)} \sum_{m=0}^{n} \operatorname{Re}\left\{\frac{\left(e^{-j \omega T}-1\right)(-1)^{m} \Gamma(\alpha+1)}{\Gamma(m+1) \Gamma(\alpha-m+1)} e^{-j m \omega T}\right\} .
$$

To evaluate the influence of the fractional derivative term, we define a new function $f(\omega)$ from the second term on the right side of inequality (8) as

$$
f(\omega)=\frac{-B_{\alpha}}{2 T^{\alpha-1}(1-\cos \omega T)} \sum_{m=0}^{n} \operatorname{Re}\left\{\frac{\left(e^{-j \omega T}-1\right)(-1)^{m} \Gamma(\alpha+1)}{\Gamma(m+1) \Gamma(\alpha-m+1)} e^{-j m \omega T}\right\} .
$$


The function can also be used to determine the maximum stiffness $K$ that can be set in the system. When $f(\omega)$ is smaller, we can set the stiffness $K$ higher. For the case of a PDVC, $f(\omega)$ is given as $f(\omega)=-B \cos (\omega T)$.

\subsection{Passivity of a single term of a fractional derivative}

We calculate the value of $f(\omega)$ for various $\alpha$. We consider the cases of $0<\alpha<1$ and $1<\alpha<$ 2 separately because the nature of $f(\omega)$ is quite different when $0<\alpha<1$ than when $1<\alpha<$ 2. Fig. 2 (a) shows the value of $f(\omega)$ for $\alpha=0.3,0.5,0.7$ and 1. Fig. 2 (b) is the value of $f(\omega)$ $\alpha=1,1.3,1.5$, and 1.7. $B_{\alpha}$ is selected as $f\left(\omega_{N}\right)=1$. In the calculation, we use a sampling period $T=2(\mathrm{msec})$, and the frequency $\omega$ is between 0 and $1570(\mathrm{rad} / \mathrm{s})$.

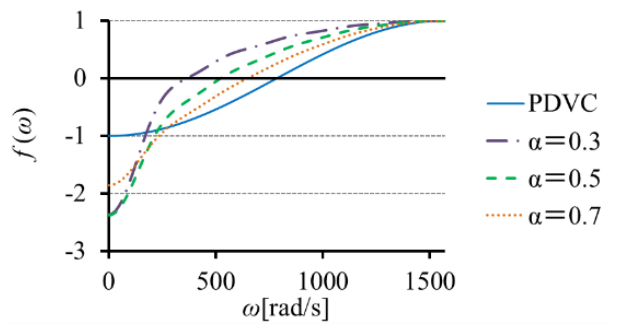

(a) $0<\alpha<1$

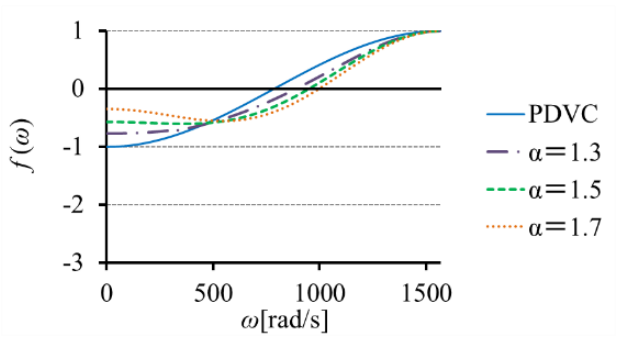

(b) $1<\alpha<2$

Fig. 2. $f(\omega)$ for a single term of a fractional derivative

In Fig. 2, the vertical axis is $f(\omega)$ and the horizontal axis is the frequency $\omega$. When $0<\alpha<1$, $f(\omega)$ increases monotonically with $\omega$, and the intersection with the $\omega$ axis moves to the right as $\alpha$ increases. As the lower value is more passive, Fig. 2 (a) shows that a $\mathrm{PD}^{\alpha} \mathrm{VC}$ with $0<\alpha<1$ is more passive in the lowest frequency range but less passive in the middle-frequency range than a PDVC. On the other hand, Fig. 2 (b) shows that when $1<\alpha<2, f(\omega)$ has a minimum, and the value of $f(0)$ increases monotonically to zero with an increasing $\alpha$. Fig. 2 (b) also shows that for $1<\alpha<2$, a $\mathrm{PD}^{\alpha} \mathrm{VC}$ is less passive in the low-frequency range but more passive in the middle-frequency range than a PDVC. By these results, it is difficult to keep better passivity in a wide range of frequency using a single term of a fractional derivative.

\section{Virtual coupling with multi terms of a fractional derivative}

\subsection{VC with a linear combination of fractional derivative terms}

Next, in order to improve the passivity in a wide range of frequency, we study a VC including multi terms of a fractional deriative, descrived by

$$
f_{a}=K x+B_{\alpha}\left(A_{1} \frac{d^{\alpha_{1} x}}{d t^{\alpha_{1}}}+A_{2} \frac{d^{\alpha_{2} x}}{d t^{\alpha_{2}}}+\cdots+A_{c} \frac{d^{\alpha_{c} x}}{d t^{\alpha_{c}}}\right)
$$

Where, $A_{d}(d=1,2, \ldots c)$ is the weight coefficient, $c$ is the number of terms of a fractional derivative. The transfer funtion and the evaluation function $f(\omega)$ are

$$
\begin{gathered}
H(z)=K+B_{\alpha} \sum_{d=1}^{c} A_{d} \frac{1}{T^{\alpha_{d}}} \sum_{m=0}^{n} \frac{(-1)^{m} \Gamma\left(\alpha_{d}+1\right)}{\Gamma(m+1) \Gamma\left(\alpha_{d}-m+1\right)} z^{-m}, \\
f(\omega)=B_{\alpha} \sum_{d=1}^{c}\left[A_{d} \frac{1}{2 T^{\alpha_{d}-1}(1-\cos (\omega T))} \sum_{m=0}^{n} \operatorname{Re}\left\{\left(1-e^{-j \omega T}\right) \frac{(-1)^{m} \Gamma\left(\alpha_{d}+1\right)}{\Gamma(m+1) \Gamma\left(\alpha_{d}-m+1\right)} e^{-j m \omega T}\right\}\right] .
\end{gathered}
$$


In (12), $f(\omega)$ depends on the the weight coefficients $A_{d}$ and the order of derivative $\alpha_{i}$.

\subsection{Optimization of the weight coefficients and the orders of fractional derivative}

In order to obtain a desired system, in which the passivity keeps lower value in a wide range, we optimize the weight coefficients and the order of derivative by using a steepest descent method. In the method, we use a evaluation function as

$$
J(\boldsymbol{x})=\sum_{d=1}^{c}\left(f\left(\omega_{d}\right)+1\right)^{2}+\left(f\left(\omega_{N}\right)-1\right)^{2}
$$

The function means that the desired value $f(\omega)$ at fixed frequencies $\omega_{d}$ is -1 , and the desired value $f(\omega)$ at the frequency $\omega_{N}$ is 1 . The last term for the $\omega_{N}$ is added for decreasing the passivity in a high-frequency range. The sequence of the steepest descent method is

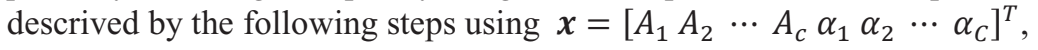

Step 1. The initial values of $\boldsymbol{x}$ are given as $\boldsymbol{x}^{(0)}$ and $k=0$.

Step 2. When Eqn. (14) is satisfied, the sequence finishes.

$$
\frac{\partial J\left(x^{(k)}\right)}{\partial x_{d}^{(k)}}<\delta \quad(d=1, \cdots, c)
$$

Step 3. The values of $\boldsymbol{x}$ is replaced by the Eqn. (15)

Step 4. Return to the step 2 with $k=k+1$.

$$
\boldsymbol{x}^{(k+1)}=\boldsymbol{x}^{(k)}-\beta\left[\frac{\partial J\left(\boldsymbol{x}^{(k)}\right)}{\partial x_{1}^{(k)}} \cdots \frac{\partial J\left(x^{(k)}\right)}{\partial x_{c}^{(k)}}\right]^{T}
$$

whrere, $\delta$ is a threshold value and $\beta$ is a positive parameter, that are decided by try and error. The result of the optimization for a $\mathrm{VC}$ with two terms of a fractional derivative was $A=[1.69$, 1.32], $\alpha=[1.50,3.47]$. Figure 3 shows $f(\omega)$ of the optimized VC.

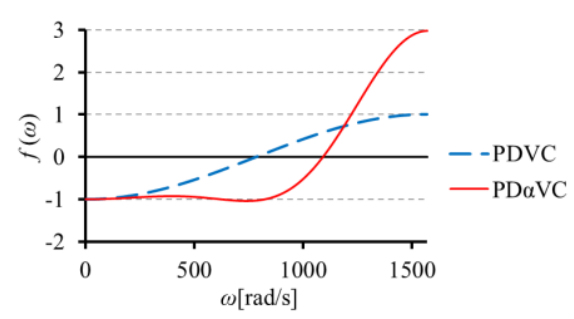

Fig. 3. $f(\omega)$ of the Optimized VC

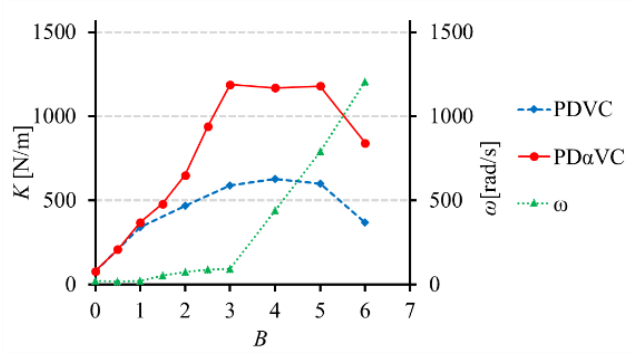

Fig. 4. Experimental results for the PDVC and the optimized VC

The figure shows that the optimized VC keeps lower passivity in the low- and middle- ranges of frequency. The passibity at high-frequency around the Nyquist frequency $\omega_{N}$, however. becomes worse.

\section{Experiment}

In order to demonstrate the effect of the optimized $\mathrm{VC}$ and the influence of the worse effect at high-frequency range, we conduct experiments. In the experiment, we use a commercialized haptic device, Geomagic Touch X. The virtual environment is a virtual floor, 
and the task is to push the floor in the vertical direction. In the experiment, we attached a small mass to the fingertip of the device, and the fingertip was dropped onto the virtual floor without any human involvement because it is difficult to repeatedly apply a force of a given magnitude to a virtual wall by human action. We measured the maximum value of $K$ for various $B_{\alpha}$. The sampling rate is $T=2$ (msec).

Figure 4 shows the experimental results for the common PDVC and the optimized VC. In Fig. 4, the solid red line corresponds to the results for the optimized VC, and the dashed blue line is the PDVC. The green line shows the occurred frequency at the experiment on the optimized VC.

\section{Conclusion}

In this research, we studied a virtual coupling including a fractional derivative approximated by a FIR approximation and assessed its characteristics. We then proposed a method to use multi terms of a fractional derivative and adjust the value of the parameters, based on a steepest descent method. Finally, we performed experiments to demonstrate the effects of the proposed virtual coupling. The analysis and experimental results show that the proposed method is effective and can be used to display virtual objects with harder surfaces.

\section{References}

1. R. J. Adams, B. Hannaford, IEEE Trans. on Robotics and Automation, 15(3), 465-474 (1999)

2. K. F. Laurin-Kovits, J. E.Colgate, S. D. R Carnes, Proc. of IEEE International Conference on Robotics and Automation, 1476-1481 (1991)

3. J. E. Colgate, J. M. Brown, Proc. of Int. Conf. on Robotics and Automation, 3205-3210 (1994)

4. J. E. Colgate, J. E. Stanley, M. C. Brown, IEEE/RSJ Proc. of the International Conference on Intelligent Robotics and Systems, 140-145 (1995)

5. M. Kawai, T. Yoshikawa, IEEE/ASME Trans. on Mechatronics, 9(1), 58-64 (2004)

6. D. W. Weir, J. E. Colgate, M. Peshkin, Symp. on Haptic Interface for Virtual Environment and Teleoperation Systems, 169-175 (2008)

7. B. S. Manohar, H. Vasudevan and M. Manivannan, Proc. of int. conf. on Haptics, 53-62 (2008)

8. J. H. Ryu, Y. S. Kim, B. Hannaford, IEEE Trans. on Robotics, 20 (4), 772-776 (2004)

9. I. Podlubny, Fractional Differential Equations, Academic Press (1999)

10. I.Petras, L.Dorcak, I. Kostial, Acta Montanistica Slovaca, 3, 143-148 (1998)

11. S. Sakakibara, JSME International Journal C, 40(3), 393-399 (1997)

12. W. Zhang, N. Shimizu, JSME International Journal C, 42(4), 1-9 (1999)

13. B. M. Vinagre, Y. Q. Chen, I. Pretras, Journal of the Franklin Institute 340, 349 -362 (2003)

14. C. Ma, Y. Hori, Trans. of the Institute of Electrical Engineers of Japan D, 124(8), 837842 (2004)

15. Y. Q. Chen, B. M. Vinagre., "A new IIR-type digital fractional order differentiator," Signal Processing, 83, 2359-2365 (2003)

16. J.A.T. Machado, Anal.-Model.-Simulation 27, 107-122 (1997)

17. A. Oustaloup, F. Levron, B. Mathieu, F. M. Nanot, IEEE Trans. on Circuits and Systems I, 47(1), 25-39 (2000) 